\title{
$\mathcal{A} \boldsymbol{C} \mathcal{A S E}$
}

or

\section{HYDATID IN THE BRAIN.}

\author{
By Mr. MICHAEL MORRAH,
}

SURGEON AT WORTHING.

COMMUNICATED BY

DR. Y E L L OL Y.

Read Feb. 12, 1811.

Elizabeth LINDUP, 19 years of age, of a most robust make, and general good health, began to complain of pain, and a swimming of her head, about three years back; which symptoms were always increased upon motion, and particularly by stooping. They continued with occasional exacerbations, accompanied by irritation of the stomach, and a suffusion of the eyes (such as is produced by crying), till the 22d of April last; when, during her occupation of cooking a dinner, she was seized, without any previous warning, with a fit; 
during which she had no convulsions, but was represented as lying motionless, her inspirations being very long and deep, and gradually becoming less so till her recovery.

This happened immediately after the completion of the menstrual flux, and lasted some minutes. I saw her before the fit was quite over, and was particularly struck with the complaint she made of an acute fixed pain of the head, and with the ferrety appearance of the eyes. One month from this time she had another fit, which seized her so unexpectedly, that she dropped with a pan of milk in her hand; and from this time till the 20th of August, she had a paroxysm every third week, which might be said to consist of two fits; one in the evening, from which she very imperfectly recovered, till after a second, which came on the next morning; after which she continued free from any fit for three weeks.

On the 20th of August, she had three fits in one day, accompanied by considerable derangement of the stomach, and by screaming, and other indications of great suffering, amounting almost to delirium. A succession of these distressing attacks increasing in severity, and with stupor intervening, continued till the middle of September, when she had nearly lost her hearing. Shortly afterwards she lost the sight of her right eye, and in fourteen days more, that of her left. Her smell was com- 
pletely gone, and the olfactory nerves were insensible to the stimulus of hartshorn; her speech and power of deglutition were very much impaired, and her left side (of which she had previously complained, as being affected with rigors) became paralytic. On Friday, the 7 th December, she fell into an apoplectic stupor, which continued till the Thursday morning following; during which period she neither spoke, nor took any nourishment. At that time she roused up, spoke, and swallowed some refreshment, but soon relapsed into the former state ; and on Friday evening, the 14th December, she died.

During the whole period, with the exception of October, she menstruated regularly. The pulse, till towards the conclusion, was not affected; there was no increase of heat; the bowels were rather costive, but easily acted upon; and the bladder did its office. At all times, however, the girl laboured under a degree of nervous irritability, unaccountable in a person of such general good health and robust organization.

On Sunday, the 16th December, I opened the head, assisted by Mr. Johnson, of the Royals. On taking off the cranium, the vessels of the dura mater appeared rather more turgid than usual. On removing the dura mater, the pia mater was seen elevated over the right hemisphere by tumor; which, on examination, was found to be a hydatid, 
about three inches long by two inches in breadth. It was imbedded in the substance of the brain, from which it was very liberally supplied with numerous minute vessels of the size of hairs, which were particularly abundant at the lower part. To its upper surface, a portion of the brain was so firmly attached by the pressure it sustained against the cranium from the increase of the hydatid, that I did not venture to attempt to separate them, and by that portion it was suspended in the glass, and sent to Mr. Cooper. The left ventricle contained a little more fluid than is usually found in a healthy subject; the right had hardly any, being compressed by the tumor. In every other respect the brain was perfect, and exhibited no morbid appearance, except the slight one of increased fulness of vessels.

Of the treatment much need not be said. It was governed by the symptoms, and was sometimes useful in moderating them; but, as must be conceived, it could have no influence in checking the progress of the disease, every period of which was clearly marked by the symptoms of oppressed brain.

Dr. Thomas Young, Physician to St. George's Hospital, saw the case occasionally with me; and gave it as his opinion, in which I fully coincided, that the symptoms arose from some organic affection of the head. 